\title{
Erythrocyte reactive nitrogen species in health and disease
}

\author{
Carlota Saldanha* and Ana S Silva Herdade \\ Instituto de Medicina Molecualr , Instituto de Bioquímica, Faculdade de Medicina, Universidade de Lisboa, Portugal
}

\begin{abstract}
In general, reactive nitrogen species, like nitric oxide (NO) and peroxynitrite are classified in primary and secondary according its benefic or deleterious effects respectively. Multiple benefic roles through the body has been attributed to nitric oxide (NO), a key signaling molecule, which acts as vasodilator relaxing smooth muscles of arteries; participates in the blood pressure control; hinders the initiation of inflammation NO liberated from endothelium cells or lymphocytes to intravascular corporal fluid enter into erythrocytes through membrane band 3 protein being after captured by deoxyhemoglobin or by oxyhemoglobin. Inside the erythrocytes several chemical reactions occur with generation of S-nitrosoglutathione a NO reservoir molecules and NO derivatives (NOx) molecules namely nitrite, nitrate and peroxynitrite. The erythrocyte acts as sensor of the tissues oxygen partial pressure and consequently change its oxygen levels lead along with NO contents. $\mathrm{NO}$ is released through band 3 protein into the blood, at microcirculatory network, in tissues with low oxygen partial pressure. The aim of this mini review was to present the erythrocyte signal transduction pathways associated with NO and S-nitrosoglutathione efflux, metabolic behavior of the NO reservoir molecules and NO reactive species in human erythrocytes under several biological conditions. For examples, the effects of external amphipathic molecules, fibrinogen, and internal manipulation of protein phosphorylation degree and redox status on erythrocyte. NOx, NO and S-nitrosoglutathione efflux levels are herein included. Erythrocyte NO efflux evidenced as biomarkers of inflammatory vascular diseases will be herein highlighted.
\end{abstract}

\section{Mini review}

Nitric oxide (NO) produced by endothelial cells and T-lymphocytes, once released into the blood circulation, can enter the erythrocyte membrane through band 3 protein [1]. Erythrocytes assure the transport of oxygen and nitric oxide obtained from the lungs under high values of oxygen partial pressure $\left(\mathrm{PaO}_{2}\right)$ to systemic tissues, with lower $\mathrm{PaO}_{2}$, where partially liberated are [2]. The primary erythrocyte function concerning the oxygenation tissue is accompanied by the mobilization of NO [3]. Thus, once NO reaches the intra-globular compartment binds to oxyhemoglobin forming the S-nitrosohemoglobin (SNO$\mathrm{Hb})$ molecules and several reactions happen, such as NO reacting with the superoxide anion forming peroxynitrite (ONOO-), which subsequently yields nitrite and nitrate [4]; all together these molecules receive the nomination of $\mathrm{NO}$ derivatives (NOx). Nitrate production in erythrocytes occurs in the presence of oxygen and oxyhemoglobin and can be converted to nitrite by the action of methemoglobin reductase [4]. It is also known that erythrocytes may release nitric oxide in the microcirculation under low oxygen tension [2]. The efflux of NO from erythrocyte occurs through a trans-nitrosylation process involving the thiol group of the band 3 protein that receives NO from SNO-Hb [4], however the precise transport mechanism is not yet clarified. The GSNO efflux from erythrocytes or red blood cells (RBCs) has also been observed by us [5]. Using fluorescence microscopy, we observed NO inside human erythrocytes after acetylcholine (ACh) stimulation and we were the first to quantify the NO efflux from erythrocytes with a potentiometer device $[4,6]$.

But until now, the majority of the published studies in the literature quantified plasma or erythrocyte nitrite but calling it NO. When NO was recognized as a vasodilator [7], we question if RBCs deformability will be affected by the presence of NO. So, using SpermineNONOate (a NO donor) and ACh as stimulator of $\mathrm{NO}$ efflux from erythrocytes, we observed an increase in RBCs deformability [8]. Opposite effects on the oxygen affinity to hemoglobin, experimentally quantified as P50, was obtained with ACh and SpermineNONOate with which we observed, respectively augmented and impairment P50 values, meaning a decrease and an increase in oxygen affinity for hemoglobin [8]. The NO donor behavior has an allosteric factor of oxygen affinity for hemoglobin, which was later confirmed [9]. Our experimental results with erythrocytes showed NO and oxygen efflux in the presence of $\mathrm{ACh}$ in opposition to $\mathrm{NO}$ influx and oxygen binding to $\mathrm{Hb}$ in the presence of a NO donor [8]. These results are identical to the ones described above about the RBCs gas transportation in tissues with high and low $\mathrm{PaO}_{2}$ [2]. As far as is known, $\mathrm{NO}$ enters into erythrocytes and turn out through membrane band 3 protein [10]. Erythrocyte membrane band 3 protein contains tyrosine residues able to become phosphorylated, by protein tyrosine kinases (PTK: p72syk or p53/56lyn), which could be dephosphorylated by protein tyrosine phosphatase (PTP). A dynamic balance between band 3 phosphorylation and dephosphorylation occurs in normal erythrocytes [11]. Both PTK and PTP enzyme activity are dependent on the protein kinase $\mathrm{C}$ (PKC) activity which phosphorylates both enzymes becoming PTP inactive and PTK in an active state [11]. So, our studies pursuit to search answers about the signal transduction mechanism associated with erythrocyte NO mobilization under influence of amphipathic and hydrophilic molecules, protein phosphorylation degree and redox status of erythrocytes.

${ }^{\star}$ Correspondence to: Carlota Saldanha, Institute of Biochemistry, Institute of Molecular Medicine, Faculty of Medicine University of Lisbon, Av. Prof. Egas Moniz, 1649-028 Lisbon, Portugal, E-mail: anarmsilva@medicina.ulisboa.pt

Key words: erythrocyte, nitric oxide, peroxynitrite, nitrite, nitrate, s-nitrosoglutathione; phosphoinositide-3 kinase, adenylyl cyclase

Received: June 20, 2018; Accepted: June 25, 2018; Published: June 30, 2018 
Nitrogen oxidative species levels and internal proteins of the human erythrocytes in health and inflammatory vascular diseases

Acetylcholine (ACh) is a ubiquitous molecule produced by endothelial cells and T lymphocytes and the mechanism of ACh resultant from endothelial cells into blood circulation was recently confirmed $[4,12]$. In erythrocyte membrane we demonstrate a cross talk between band 3 protein and acetylcholinesterase (AChE) enzyme binding to its natural substrate, $\mathrm{ACh}$ or to an inhibitor, velnacrine maleate (VM) [13]. It was also confirmed that the active AChE- ACh and inactive $\mathrm{AChE}-\mathrm{VM}$ complexes conformations associated with adenylyl cyclase through $\mathrm{G}_{\mathrm{i}} \alpha \beta$ protein result in lower adenylyl cyclase (cAMP) production [13]. It was also shown that both enzyme complex conformations activate protein kinase $\mathrm{C}$ (PKC) which in turn inhibits, by phosphorylation, the phosphodiesterase -3 (PDE-3), without changes on cAMP concentration [14].

When AChE-ACh enzyme complex occurs in RBCs membrane, high levels of NO efflux, nitrite and nitrate concentrations were observed in the absence or presence of band 3 phosphorylation induced by calpeptin, the inhibitor of PTP $[4,14]$. Although, the GSNO levels are independent of band 3 phosphorylation degree [14].

Higher significant levels of NO efflux from RBCs were achieved when band 3 is dephosphorylated by the p72syk inhibitor in the presence of the inactive enzyme complex AChE-VM, when compared to the AChE-ACh complex [14].

Regarding the nitrite and nitrate levels they are independent of band 3 phosphorylation degree when the AChE-VM inactive complex is present in the RBCs membrane [14].

Both PTK and PTP enzymes are also phosphorylated by protein kinase C (PKC) resulting in PTP inactivation and PTK activation [11]. When $\mathrm{RBCs}$ present a higher band 3 protein phosphorylation status, this is followed by an increased AChE enzyme activity [13].

When the band 3 protein phosphorylation results from the presence of a PKC activator, high values of $\mathrm{NO}$ efflux are obtained with AChE$\mathrm{ACh}$, at variance of lower values verified under PKC inhibition $[5,15]$. These results reinforce the association between band 3 phosphorylation and NO efflux from erythrocytes and confirm the role of cAMP levels in the signal transduction pathway; its involvement is dependent of PKC activators or inhibitor concentration by direct manipulation in erythrocytes.

The NO efflux from erythrocytes measured in the presence of AChE-VM was lower in relation to those determined in the presence of AChE-ACh [14]. In erythrocytes the inactive AChE-VM enzyme complex yield lower nitrite and nitrate levels than those quantified with AChE-ACh complex [4,14].

We verified decreased amounts of GSNO and peroxynitrite inside erythrocytes when, on its membrane, AChE-ACh active complex was present, in relation to the values obtained when the inactive complex ACh-VM is formed [14]. Lower GSNO concentration in erythrocytes may result from lower glutathione (GSH) content. Reduced free GSH inside RBCs can be a consequence of lower reduced potential in the form of nicotinamide adenine dinucleotide phosphate concentration, a biomarker of the lower oxidative phase of phosphopenthose pathway; or from the existence of GSNO efflux from erythrocytes. We have shown that no variations are induced by both AChE-ACh and ACHEVM complexes in glucose-6-phosphodehydrogenase, glyceraldehyde- 3-phosphate dehydrogenase enzyme activities neither on 2,3bisphosphoglycerate and L-lactate concentrations [14].

We are the former to measure GSNO liberated by erythrocytes in the presence of ACh [5]. So, the complex AChE-ACh induces GSNO efflux and contributes to lower the levels inside erythrocyte in relation to those obtained with the inactive complex AChE-VM [5,14].

When changes on AChE molecular conformations were induced by antibodies against the $\mathrm{C}$ or $\mathrm{N}$ terminus decreased amounts of GSNO and NO efflux from erythrocytes were obtained [13]

The variation of erythrocyte NO efflux, peroxynitrite and GSNO levels observed in the presence of velnacrine and ACh called our attention to investigate the NO metabolism in a set of redox status modulation. At that time, we studied the effects of dithiothreitol (DTT; a thiol reducing compound) to function as a thiol (SH) donor. It is known that $\mathrm{SH}$ groups are easily kept in the reduced state once a second thiol is added, such as cysteine or glutathione because they rapidly exchange disulfide groups. RBCs in the presence of DTT show significantly internal NO dependent reactions originating an increase in nitrite, nitrate and GSNO concentrations and a decrease on the NO efflux from erythrocytes. Peroxynitrite levels remained unchanged with AChE-ACh or AChE - VM enzyme complexes in the presence of DTT, but the levels of nitrite and nitrate molecules decreased. Significantly increased peroxynitrite concentrations were found in RBCs AChEVM plus DTT $1 \mu \mathrm{M}$ and plus DTT $50 \mu \mathrm{M}$ but lower plus DTT $10 \mu \mathrm{M}$ [16]. We concluded that dithiothreitol induces activation of erythrocyte thiol status by decreasing NO efflux and allowing intracellular NO mobilization into different derivative molecules, both in the absence and presence of the AChE-ACh and AChE-VM enzyme complexes [16].

Beyond the hemostasis properties of fibrinogen (Fib), this protein is a hemorheological promoter of RBCs aggregation and plasma viscosity [17]. Fibrinogen is also known as one of the acute phase protein associated with inflammatory conditions [18]. Fib binds to erythrocyte CD47 and at a normal range of concentrations is able to decrease NO efflux $[19,20]$.

But high levels of Fib, observed in inflammatory diseases, have shown an increase in NO efflux from RBCs in dependence of the band 3 protein phosphorylation and lower levels of cAMP [20] (Figure 1).

Previously we verify an increased AChE enzyme activity in patients with glaucoma under timolol maleate therapy for reducing increased intra-ocular pressure and we evidenced that timolol is a weak inhibitor of erythrocyte AChE [21]. When the less active complex AChE-timolol is formed in erythrocytes obtained from healthy donors, lower NO efflux and lower S-GSNO levels in erythrocytes compared with the active complex AChE-ACh were found [22]. The RBCs of glaucoma patients without timolol therapy are able to liberate the same amount of NO and have equal GSNO levels that are obtained after incubation with timolol [23].

\section{Erythrocyte NO availability as a marker of inflammatory vascular diseases}

When the less active complex AChE-timolol is formed in erythrocytes obtained from glaucoma patients higher NO efflux and GSNO content were observed in relation to those assessed in RBCs from healthy humans [23]. In this painful and progressive ocular disease the decrease ability to preserve NO inside the RBCs may be a deleterious nitrogen reactive species contributing to worse the vascular endothelial dysfunction. 


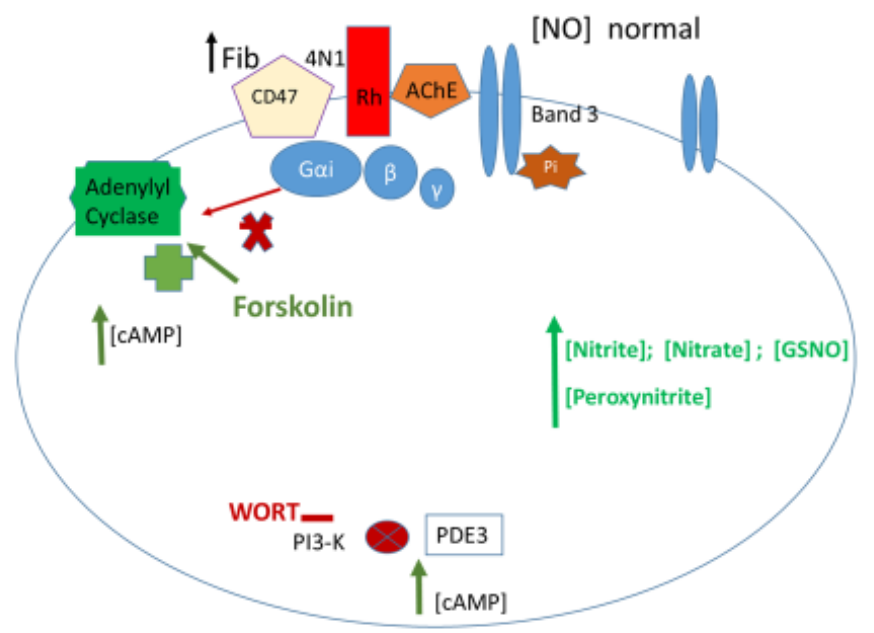

Figure 1. Representative diagram of normal $\mathrm{NO}$ efflux from erythrocyte in an in vitro model of hyperfibrinogenemia. Fibrinogen (Fib) binds to CD47. It was shown that the amount of NO efflux is increased at lower adenosine monophosphate (cAMP) and normalized at high cAMP levels achieved in presence of forskolin and wortamannin (WORT), respectively activator of adenylyl cyclase and inhibitor of phosphodiesterase (PDE3)

In two groups of patients' one with systemic lupus erythematosus and the other with rheumatoid arthritis, characterized by subclinical atherosclerosis, the NO efflux values obtained from their erythrocytes associate negatively with carotid intima-media thickness (cIMT) and with the presence of plaques and NO efflux is an independent predictor of cIMT [24]. In these immune diseases NO may be considered a primary nitrogen reactive species resulting from its rescue inside the RBCs.

Another painful and mortal disease in small years after appearance with very rare exceptions is amyotrophic lateral sclerosis (ALS) [25]. RBCs of these patients showed higher AChE enzyme activity, lower nitrite concentrations and lower NO efflux from RBCs than those obtained in healthy humans; higher NO quartiles values associated with worse respiratory function; positive relation between quartile values were obtained between AChE enzyme activity and nitrites [25]. The discrepancy between the active state of AChE enzyme and lower NO efflux observed in the blood samples of the ALS patients must be explored in future studies. But we hypothesized that these biochemical parameters can be considered as biomarkers of ALS disease [25].

Also in sepsis, some studies have regarded the erythrocyte NO availability. But besides a lot of research that has been done in all classes of sepsis it is insufficient due to the high level of mortality of this disease. We verify in a follow-up study conducted in an intensive care unit (ICU) that patients with septic shock, that died, showed higher efflux of NO from RBCs than the survivors. The NO may combine with oxygen anion peroxide produced by the dysfunctional vascular endothelium originating peroxynitrite; this is a secondary nitrogen reactive species with deleterious effects on all blood components and a marker of inflammation. Moreover, blood flow and high microvascular flow index were observed in those septic patients before dead with a device to follow and measured the hemodynamic parameters in sublingual microcirculation [26].

\section{Conclusions}

The erythrocyte nitrogen reactive species were evidenced to be dependent on circulating acetylcholine, fibrinogen and inhibitors of acetylcholinesterase, redox thiol status, protein degree phosphorylation and cAMP levels. Erythrocyte NO efflux and AChE may be considered as biomarkers of inflammatory vascular diseases.

\section{Acknowledgments}

This work was funded by Fundação para a Ciência e Tecnologia: LISBOA-01-

0145-FEDER-007391, project cofunded by FEDER, through POR Lisboa 2020 - Programa Operacional Regional de Lisboa, PORTUGAL 2020. The authors thanks Teresa Freitas and Emilia Alves for their technical and type-writing support respectively.

\section{References}

1. Huang KT, Han TH, Hyduke DR, Vaughn MW, Van Herle H, et al. (2001) Modulation of nitric oxide bioavailability by erythrocytes. Proc Natl Acad Sci U S A 98: 1177111776. [Crossref]

2. Singer M, Nathan, AT (1999) The oxygen trail: Tissue oxygenation. British Medical Bulletin 55: 96-108.

3. Stamler JS, Jia L, Eu JP, McMahon TJ, Demchenko IT, et al. (1997) Blood flow regulation by S-nitrosohemoglobin in the physiological oxygen gradient. Science 276 2034-2037. [Crossref]

4. Carvalho FA, Mesquita R, Martins-Silva J, Saldanha C (2004) Acetylcholine and choline effects on erythrocyte nitrite and nitrate levels. J Appl Toxicol 24: 419-427. [Crossref]

5. Teixeira P, Napoleão P, Saldanha C (2015) S-nitrosoglutathione efflux in the erythrocyte. Clin Hemorheol Microcirc 60: 397-404. [Crossref]

6. Carvalho FA, Martins-Silva J, Saldanha C (2004) Amperometric measurements of nitric oxide in erythrocytes. Biosens. Bioelectron 20: 505-508.

7. Vanhoutte PM (1989) Endothelium and control of vascular function. State of the Art lecture. Hypertension 13: 658-667. [Crossref]

8. Mesquita R, Pires I, Saldanha C, Martins e Silva J (2001) Effects of acetylcholine and SpermineNONOate on erythrocyte hemorheologic and oxygen carrying properties. Clin. Hemorheol. Microcirc 25: 153-163.

9. Stepuro TL, Zinchuk VV (2006) Nitric oxide effect on the hemoglobin-oxygen affinity. J Physiol Pharmacol 57: 29-38. [Crossref]

10. Saldanha C, Silva-Herdade A.S (2014) The Ubiquity Nature of Acetylcholine Clinical \& Experimental Pharmacology 4: 1-10.

11. de Oliveira S, Saldanha C (2010) An overview about erythrocyte membrane. Clin Hemorheol Microcirc 44: 63-74. [Crossref]

12. Wilson C, Lee MD1, McCarron JG1 (2016) Acetylcholine released by endothelial cells facilitates flow-mediated dilatation. J Physiol 594: 7267-7307. [Crossref]

13. Carvalho FA, de Almeida J, Freitas-Santos T, Saldanha C (2009) Modulation of erythrocyte acetylcholinesterase activity and its association with $\mathrm{G}$ protein-band 3 interactions. J Membr Biol 228: 89-97.

14. Carvalho FA, Almeida JP, Fernandes IO, Freitas-Santos T, Saldanha C (2008) Nonneuronal cholinergic system and signal transduction pathways mediated by band 3 in red blood cells. Clin Hemorheol Microcirc 40: 207-227.

15. Teixeira P, Duro N, Napoleão P, Saldanha C (2015) Acetylcholinesterase conformational states influence nitric oxide mobilization in the erythrocyte. J Membrane Biol 248: 349354

16. Lopes de Almeida P, Carvalho FA, Silva-Herdade AS, Santos-Freitas T, Saldanha C (2009) Redox thiol status plays a central role in the mobilization and metabolism of nitric oxide in human red blood cells. Cell Biol Int 33: 268-275.

17. Rampling MW, Meiselman HJ, Neu B, Baskurt OK (2004) Influence of cell-specific factors on red blood cell aggregation. Biorheology 41: 91-112. [Crossref]

18. Kushner I (1982) The phenomenon of the acute phase response. Ann N Y Acad Sci 389: 39-48. [Crossref]

19. de Oliveira S, Vitorino de Almeida V, Calado A, Rosário HS, Saldanha C (2012) Integrin-associated protein (CD47) is a putative mediator for soluble fibrinogen interaction with human red blood cells membrane. Biochim Biophys Acta 1818: 481-90.

20. Carlota Saldanha M, Ana S Silva-Herdade (2018) Fibrinogen Involvement in Hemorheology and Inflammation Advances in Medicine and Biology. In: Leon V, (Eds) Berhardt Series: Advances in Medicine and Biology Binding: Hardcover 128 115-146. 
21. Zabala L, Saldanha C, Martins e Silva J, Souza Ramalho P (1999) Red blood cell membrane integrity in primary open angle glaucoma: ex vivo and in vitro studies. Eye 13: $101-103$.

22. Saldanha C, Teixeira P, Santos-Freitas T, Napoleão P (2013) Timolol modulates erythrocyte nitric oxide bioavailability J Clin Exp Optalmol 4: 1-10.

23. Esteves R, Freitas T, Teixeira P, Napoleão P, Neves C, et al. (2016) Erythrocyte nitric oxide in glaucoma patients - ex vivo study. Clinic Hemorheol Microcirc 64: $989-$ 994
24. Fonseca J, Santos MJ, Mendes P, Canhão H, Fernandes J, et al. (2011) Hemorheological parameters are related to subclinical atherosclerosis in systemic lupus erythematosus and rheumatoid arthritis patients. Atherosclerosis 219: 821-826.

25. Lima C, Pinto S, Napoleão P, Pronto Laborinho AC, Barros MA, et al. (2016) Identification of erythrocyte biomarkers in amyotrophic lateral sclerosis. Clin Hemorheol Microcirc 63: 423-437.

26. Saldanha C, Oliveira I, Napoleão P (2017) Biophysical role of red blood cells in microcirculation. J Vas Res 54: 60-67.

Copyright: (C2018 Saldanha C. This is an open-access article distributed under the terms of the Creative Commons Attribution License, which permits unrestricted use, distribution, and reproduction in any medium, provided the original author and source are credited. 